\title{
A Research on Front-End Garbage Classification Based on Machine Vision
}

\author{
Longyu Gao*, Zhiqing Xiao, Junlong Hao, Luqi Shen, Manqian Hu \\ Tianjin University of Science and Technology, China; \\ E-mail:*ggafcc@163.com \\ www.tust.edu.cn
}

\begin{abstract}
Adding a machine vision recognition module to the traditional smart trash can can effectively improve the efficiency of trash recognition. The intelligent garbage classification model constructed by the convolutional neural network can accurately identify the types of garbage, with an average accuracy rate of 0.87 . Deploy the trained model on openMV and test it on the produced physical trash can. After the system is stable, the average time to complete a sorting and recovery is 2 s. Experiments show that the system can effectively identify the types of garbage and complete garbage classification and recycling.
\end{abstract}

Keywords: machine vision, intelligent classification, convolutional neural network

\section{Introduction}

With the rapid economic development and the continuous improvement of people's living standards, the amount of garbage has increased sharply in the cycle of consumption and production. According to the research report, by 2050 , the global waste volume will increase by $70 \%$, from 2.01 billion tons in 2016 to 3.4 billion tons in 2050. The task of sorting waste is very difficult, and it is already very difficult to study an effective method for sorting waste. urgent. Scholars at home and abroad have done a lot of analysis on waste classification, but most of the proposals put forward are innovations in terminal recycling methods. Front-end collection still relies on people's consciousness, and the accuracy of garbage classification is very low. Research on an effective front-end collection method is of great significance to the status quo of garbage classification.

For the front-end collection of garbage, smart trash cans have begun to appear in foreign markets, such as Nastar in the United States, ccko (cckoing) in Germany, Zarton and somatosensory smart trash cans in Japan, which are mostly designed based on infrared sensors and LCD screens. Domestic research has also been carried out to make trash cans have functions such as automatic classification, alarming and monitoring of available capacity, but it has not yet been able to meet the needs of automatic identification and classification.

With the rapid development of artificial intelligence technology, machine vision technology has gradually matured, and image classification methods based on deep learning have gradually diversified. This provides a new direction for the research on garbage recognition in this article. Scholars have made a lot of efforts in garbage recognition and image classification. Research and achieved certain results. For example, using the YOLOv4 network for garbage identification, a detection accuracy of $94.8 \%$ for dry and wet garbage can be achieved, and an accuracy rate of over $90.0 \%$ can be achieved in a complex detection environment.

This paper proposes an automatic garbage classification system based on machine vision. The system can meet the classification requirements of recyclables, kitchen waste, other waste and hazardous waste. The intelligent garbage classification model constructed by the convolutional neural network can accurately identify the types of garbage. With mechanical structures such as the front and rear push

(C) The 2021 International Conference on Artificial Life and Robotics (ICAROB2021), January 21 to 24, 2021 
rods and left and right flaps, the entire garbage classification process can be completed.

\section{Overall system design}

Using Solidworks software to model the trash can, the overall structure is shown in Figure 1. The trash can includes a box body, the box body has a top plate, a camera is installed on the lower surface of the top plate, and the box body is provided with a plurality of sorting compartments for collecting different types of garbage. The sorting compartments are arranged oppositely to form two Row ${ }^{1}$; the upper middle of the sorting compartment is provided with a garbage flipping plate that can be driven left and right to flip, the garbage flipping plate is a trough-shaped structure that can accept garbage, and the trough-shaped structure is provided with the garbage to be accepted Push the push plate toward the front. The structural design of the trash can is shown in Figure 1.

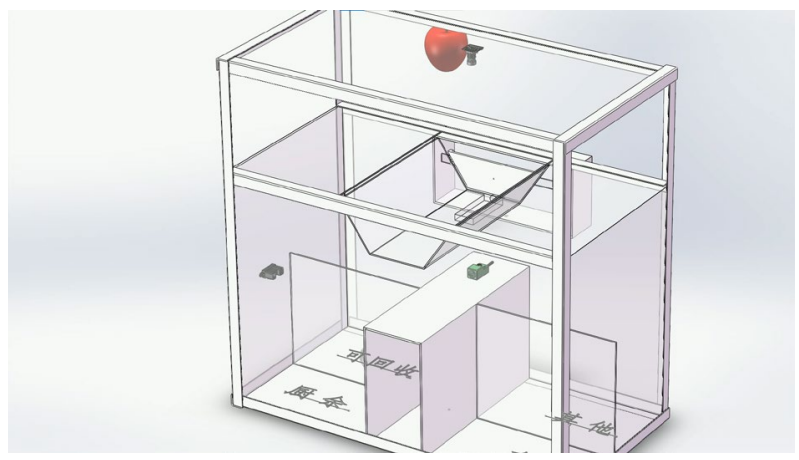

Fig.1. Structural design of trash can

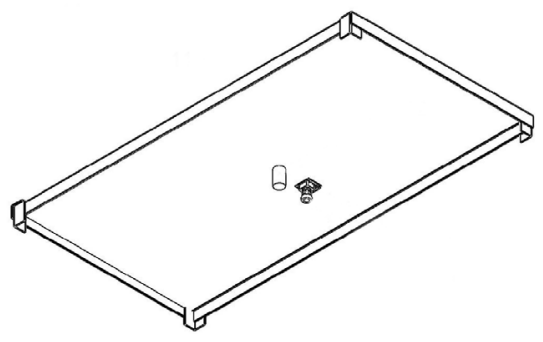

Fig.2. Roof structure

A light sensor and a light source module are also installed in the identification barrel, which are located in the top cover part, which is shown in FIG. 2. To provide a light source for machine vision at night, the light sensor and the light source module are respectively connected to the central processing module, and the light sensor is used for sensing and recognizing light changes in the barrel; the light source module is used for providing the light source for the machine vision module.

The inner wall of the trash can is equipped with an infrared sensor. When the trash is thrown in, the infrared sensor emits and receives the infrared rays reflected by the object, which can detect the filling height of the trash.

\subsection{Master chip}

This text selects STM32f407zet6 as the core controller of the hardware control system, which is mainly used to realize the functions of data acquisition and analysis and execution of component motion control. The STM32f407zet6 processor is an Arm Cortex-M4 high-performance core with a maximum operating frequency of $168 \mathrm{mHz}$ and a package form of 1qfp144. Its peripheral configuration is powerful, supporting communication interfaces such as SP interface, I2C interface, USB interface, USART interface, and peripherals such as ADC and timer. The development board used is connected to OpenMV, and OpenMV obtains garbage pictures, processes the obtained picture information, and recognizes the types of them. Finally, return the identified garbage type information to the STM32f407zet6 development board ${ }^{2}$. The development board controls the movement of the steering gear to realize the movement of the front and rear push rods and the left and right flaps, and then the garbage is dropped into the correct box. STM32F103ZET6 microcontroller is shown in Figure 3.

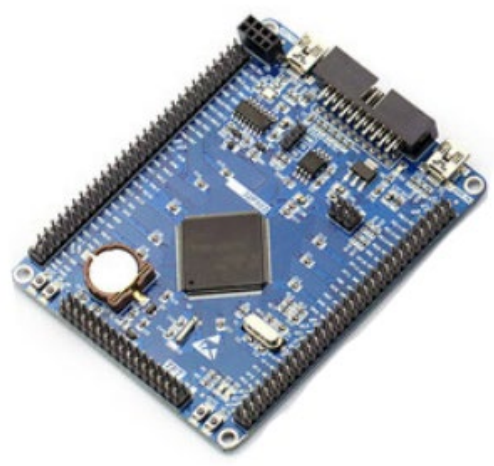

Fig.3. STM32F103ZET6

(C) The 2021 International Conference on Artificial Life and Robotics (ICAROB2021), January 21 to 24, 2021 


\subsection{OpenMV}

The vision part uses OpenMV single-chip microcomputer to communicate with STM32. The OpenMV camera is a small, low-power, low-cost circuit board that can be scripted by a high-level language Python (to be precise, MicroPython). You can use an external terminal to trigger shooting or execute an algorithm, or use the result of the algorithm to control the IO pin $^{3}$.

OpenMV uses STM32H743II ARM Cortex M7 processor, $480 \mathrm{MHz}, 1 \mathrm{MB}$ RAM, $2 \mathrm{MB}$ flash. All I/O pins output $3.3 \mathrm{~V}$ and are $5 \mathrm{~V}$ tolerant. The OpenMV module is shown in Figure 4.

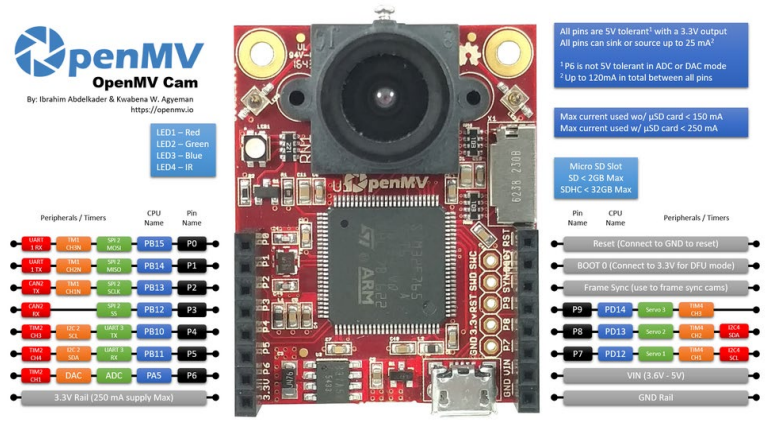

Fig.4. OpenMV module

\subsection{DS5160 Servo}

The smart trash can passes through the DS5160 servo. The working voltage of the steering gear is $6 \mathrm{~V}-8.4 \mathrm{~V}$, and the controllable angle is $180^{\circ}$. The DS5160 actuator works fast and has stable performance. Its control method is PWM control, pulse width range: 500-2500us, control precision: 3 us. The actuator can quickly respond to the motion instructions of the system and sort different types of garbage into the corresponding bins. The DS5160 servo is shown in Figure 5.

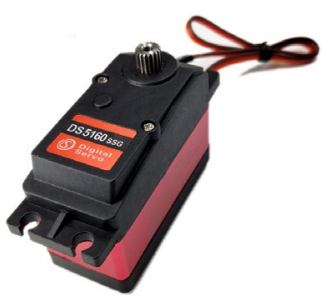

Fig.5. DS5160 Servo

\subsection{Classification agency}

This intelligent sorting trash can realizes garbage classification based on machine vision by using the camera. Through the left-right flip of the garbage dumping plate and the front and back movement of the push plate, it can realize the movement of the garbage in two dimensions, and then realize the movement of the garbage position, and finally realize The correct classification of garbage is sorted and placed in the classification cabin below to complete the automatic classification of garbage. The classification mechanism module is shown in Figure 6.

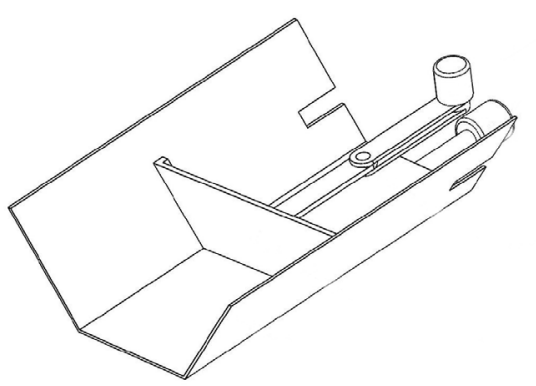

Fig.6. Classification mechanism module

\subsection{Overflow reminder module}

In order to facilitate the frequent overflow of garbage in the trash bin, the system is designed to detect the full bin. By installing an ultrasonic module just above each sorting bin, periodically detecting the distance L1 between the garbage in the bin and the module, and making the difference with the distance L2 from the module to the bottom of the sorting bin, you can get the current height $\mathrm{H}$ of the garbage in the sorting bin, that is, $\mathrm{H}=\mathrm{L} 2$-L1. If $\mathrm{H}$ exceeds $90 \%$ of the height of the sorting bin, the mark is that the sorting bin is full. When the bin is full for processing, a reminder will be sent to the corresponding APP through the WiFi module to remind relevant personnel to clean up the garbage in the bin as soon as possible. On the other hand, in order to avoid increasing the burden, the Raspberry Pi should disable the recognition function, that is, put it aside for processing before the full bucket state is removed, and no longer run the neural network to identify the thrown garbage until the full bucket state is removed. 




Fig.4. Capacitive proximity sensor

\subsection{Analysis of results}

This verification experiment conducted experiments on 6 commonly used types of garbage. For each type of garbage, 10 representatives with obvious differences in appearance were selected, and 100 throws were made at different angles, sizes, and different degrees of damage. The result data of mechanical structure classification was recorded. The specific data records are shown in Table 1.

It can be seen from Table 1 that this system has an accuracy rate of over $87 \%$ for the identification of common 6 types of garbage in actual use, and from the appearance model of the trash can in this experiment, it can be seen that the size of the trash can is small and meets the expected design requirements. It is also possible to increase the types of garbage identification by expanding the training set. The trash can realizes the two-dimensional movement of trash through the movement of the front and rear push rods and the left and right flaps, thereby realizing the correct classification of trash. The average time for each classification is about 2 seconds, which meets the experimental expectations. The system identification data table is shown in Table 1.

Table 1. System identification data table

\begin{tabular}{lll}
\hline Type of garbage & $\begin{array}{l}\text { Recognition correct } \\
\text { times/time }\end{array}$ & Average recognition rate \\
\hline Can & 87 & $87 \%$ \\
plastic bottle & 92 & $92 \%$ \\
Milk carton & 90 & $90 \%$ \\
Paper cup & 94 & $94 \%$ \\
Paper ball & 93 & $93 \%$ \\
battery & 95 & $95 \%$ \\
\hline
\end{tabular}

\section{Conclusion}

This article mainly studies the intelligent classification of urban trash bins based on machine vision, uses convolutional neural networks to build an intelligent trash classification model, and then realizes real-time monitoring of the filling status of smart trash bins through the Internet of Things technology to achieve intelligent control of trash bins .

The convolutional neural network is applied to garbage classification, the design scheme is simple to implement, and the research results achieve practical results. At present, there are very few trash cans that are classified in the process of centralized garbage collection on the market. The innovation of this implementation scheme lies in the combination of new convolutional neural network technology, which can transform ordinary trash cans into smart devices through low-cost embedded devices. Trash can to realize the function of identifying and automatically sorting garbage.

\section{References}

1.Golnabi, H., A. Asadpour. Design and application of industrial machine vision systems. Robotics and Computer-Integrated Manufacturing, 2007, 23(6): pp. 630-637.

2.Liu, Jianghai, Yadong Jiang. Design of intelligent trash can be based on machine vision. 2020 International Conference on Image, Video Processing and Artificial Intelligence, Vol. 11584. International Society for Optics and Photonics, 2020.

3.Vernon, David. Machine vision-Automated visual inspection and robot vision. STIA 92 (1991): 40499. 